\title{
Convergent Synthesis of the Quinolone Substructure of BILN 2061 via Carbonylative Sonogashira Coupling/Cyclization
}

\author{
Nizar Haddad*, Jonathan Tan and Vittorio Farina
}

\section{Supporting Information}

\section{General Experimental Methods}

${ }^{1} \mathrm{H}$ NMR spectrum of compound 4

${ }^{13} \mathrm{C}$ NMR spectrum of compound 4

${ }^{1} \mathrm{H}$ NMR spectrum of compound 7

${ }^{1} \mathrm{H}$ NMR spectrum of compound $\mathbf{8}$

${ }^{13} \mathrm{C}$ NMR spectrum of compound $\mathbf{8}$

${ }^{1} \mathrm{H}$ NMR spectrum of compound $\mathbf{1 1}$

${ }^{13} \mathrm{C}$ NMR spectrum of compound $\mathbf{1 1}$

${ }^{1} \mathrm{H}$ NMR spectrum of compound 9

${ }^{13} \mathrm{C}$ NMR spectrum of compound 9

${ }^{1} \mathrm{H}$ NMR spectrum of compound 5

${ }^{13} \mathrm{C}$ NMR spectrum of compound $\mathbf{5}$

${ }^{1} \mathrm{H}$ NMR spectrum of compound $\mathbf{2}$

${ }^{1} \mathrm{H}$ NMR spectrum of compound 2 (Authentic Sample)

${ }^{13} \mathrm{C}$ NMR spectrum of compound 2

${ }^{13} \mathrm{C}$ NMR spectrum of compound 2 (Authentic Sample)
Table of Contents

Page S2

Page S3

Page S4

Page S5

Page S6

Page S7

Page S8

Page S9

Page S10

Page S1 1

Page S12

Page S13

Page S14

Page S15

Page S16

Page S17 


\section{General Experimental Methods:}

Unless otherwise specified, all reactions were carried out in oven-dried glassware under an atmosphere of nitrogen. NMR spectra were recorded on a 400 NMR spectrometer. Shifts are reported in ppm relative to tetramethylsilane; coupling constants $(J)$ are reported in Hertz, and refer to apparent peak multiplicities. Commercially available starting materials and dry solvents were used as received without further purification. Flash chromatographic separations were carried out on $230-400$ mesh silica gel 60. HRMS were performed by positive electro-spray ionization. 
${ }^{1} \mathrm{H}$ NMR spectrum of compound 4 .

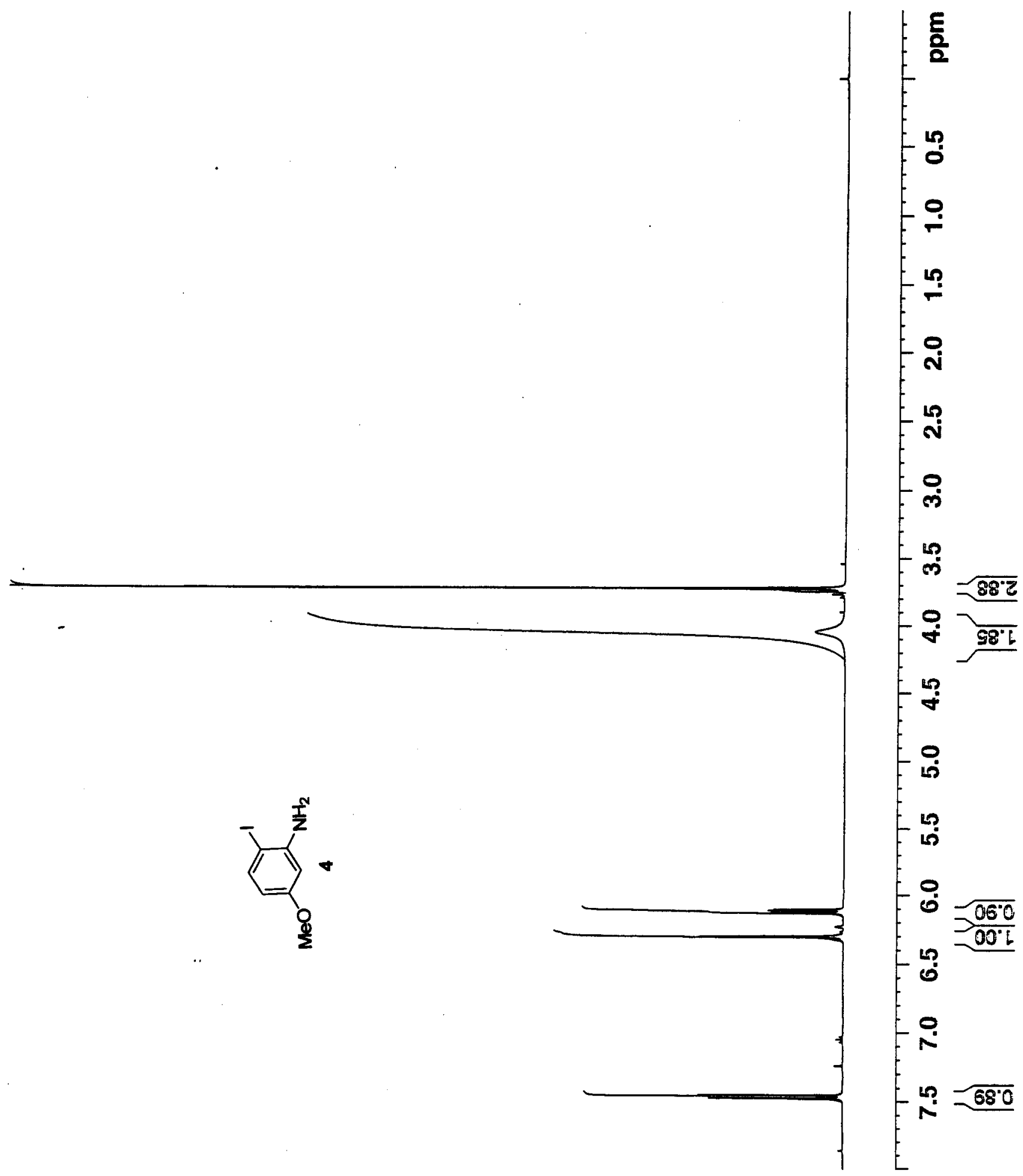


${ }^{13} \mathrm{C}$ NMR spectrum of compound 4.

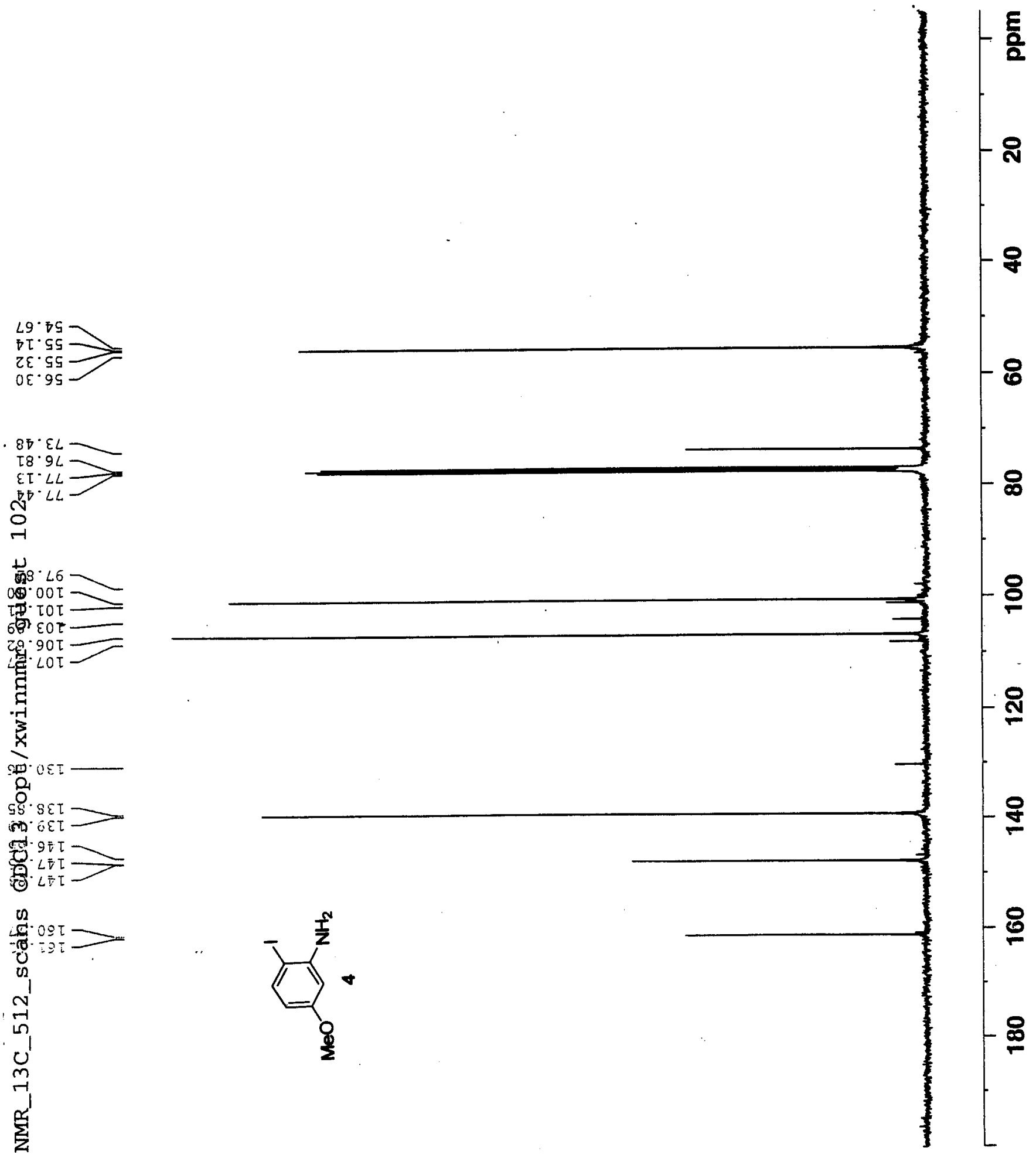




\section{${ }^{1} \mathrm{H}$ NMR spectrum of compound 7}

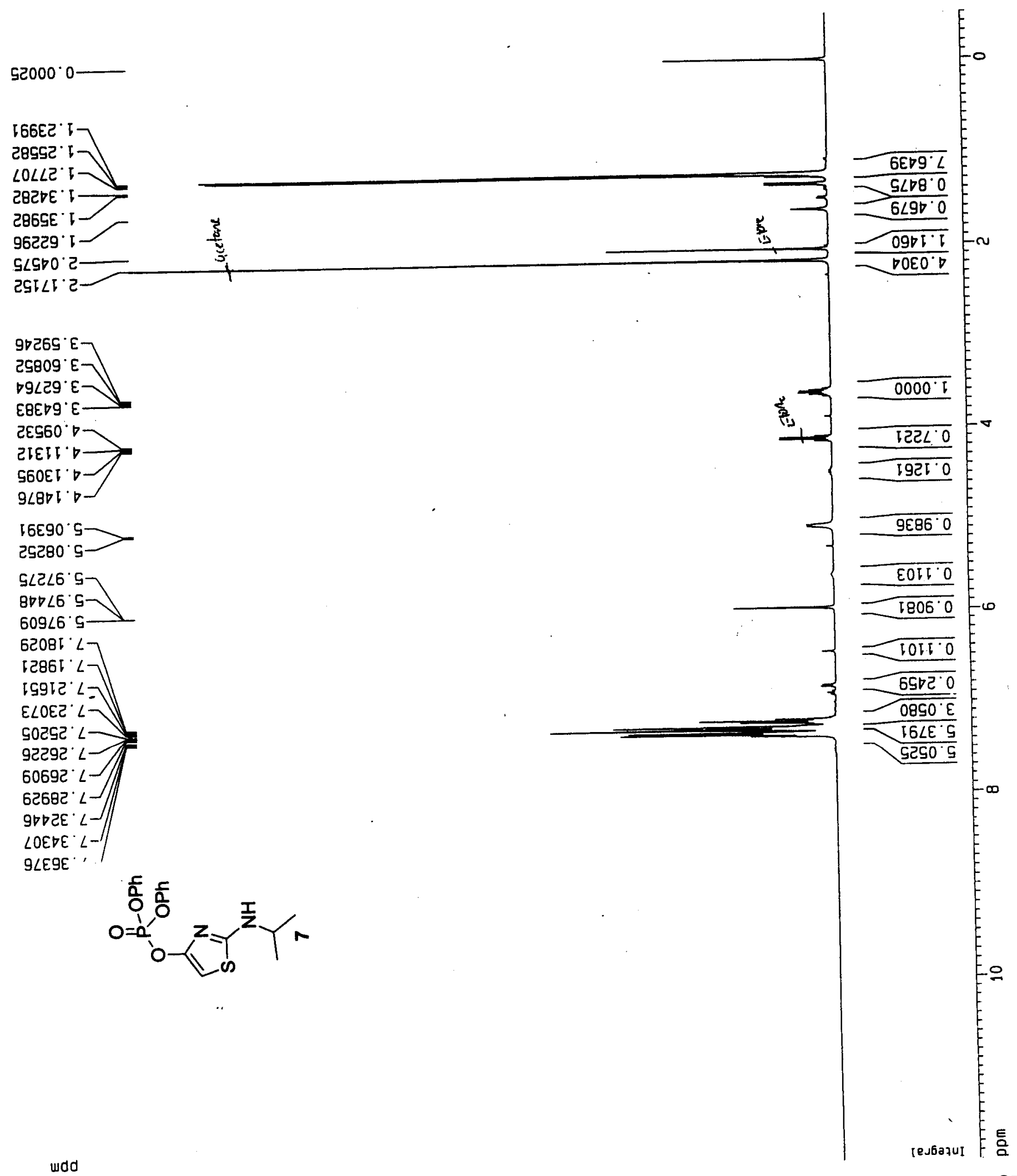




\section{${ }^{1}$ H NMR spectrum of compound 8}

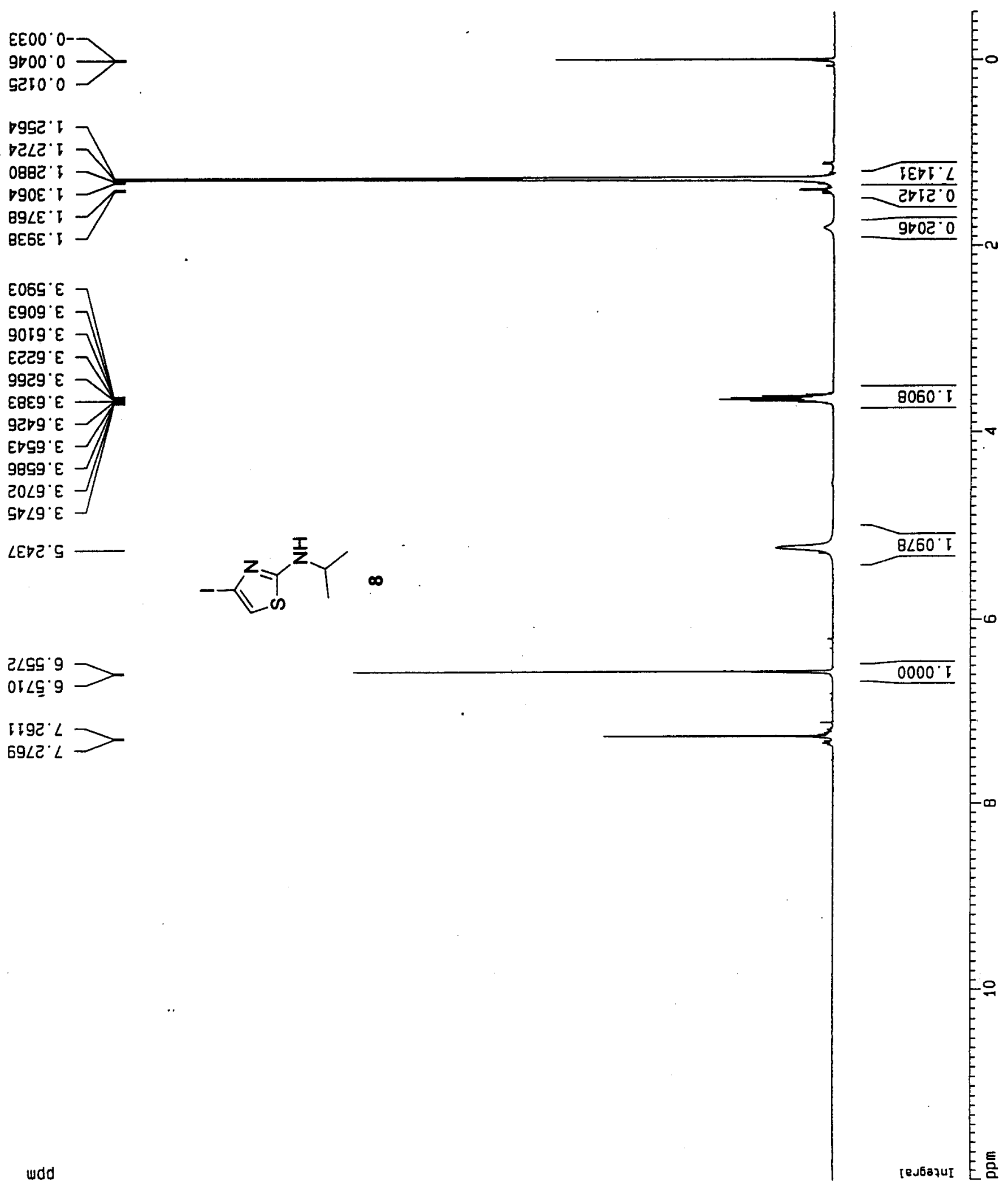




\section{${ }^{13} \mathrm{C}$ NMR spectrum of compound 8}

$D 2 L$

$\checkmark 80^{\circ} 87$

$969^{\circ} 9 L$

IEE $\angle L$

$000^{\circ} 06$

$9 \nabla L 605$

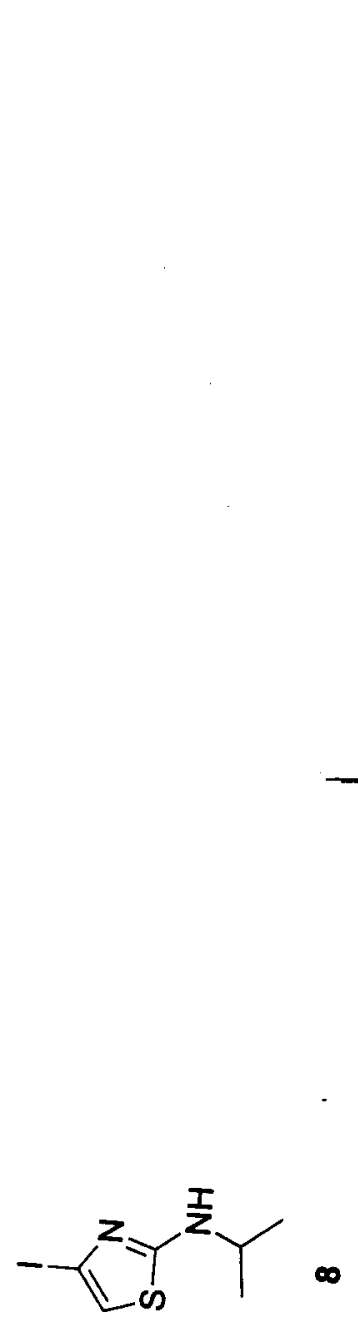

$989 \cdot 691-$
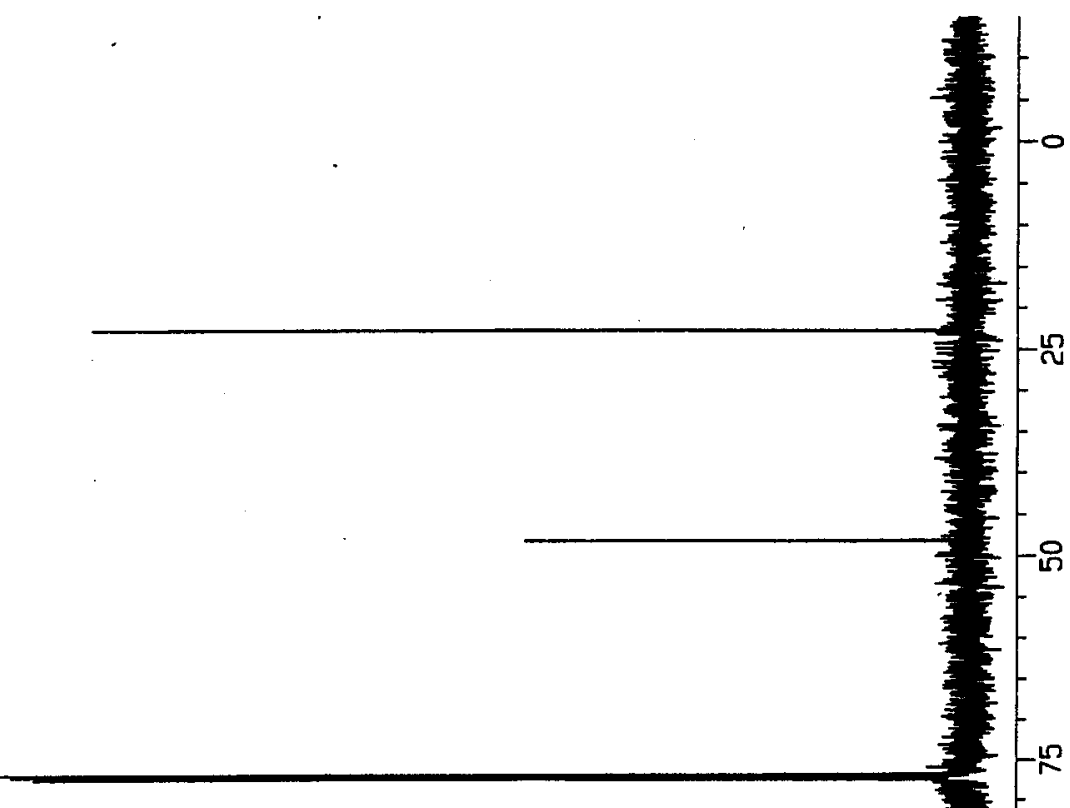
${ }^{1} \mathrm{H}$ NMR spectrum of compound 11.

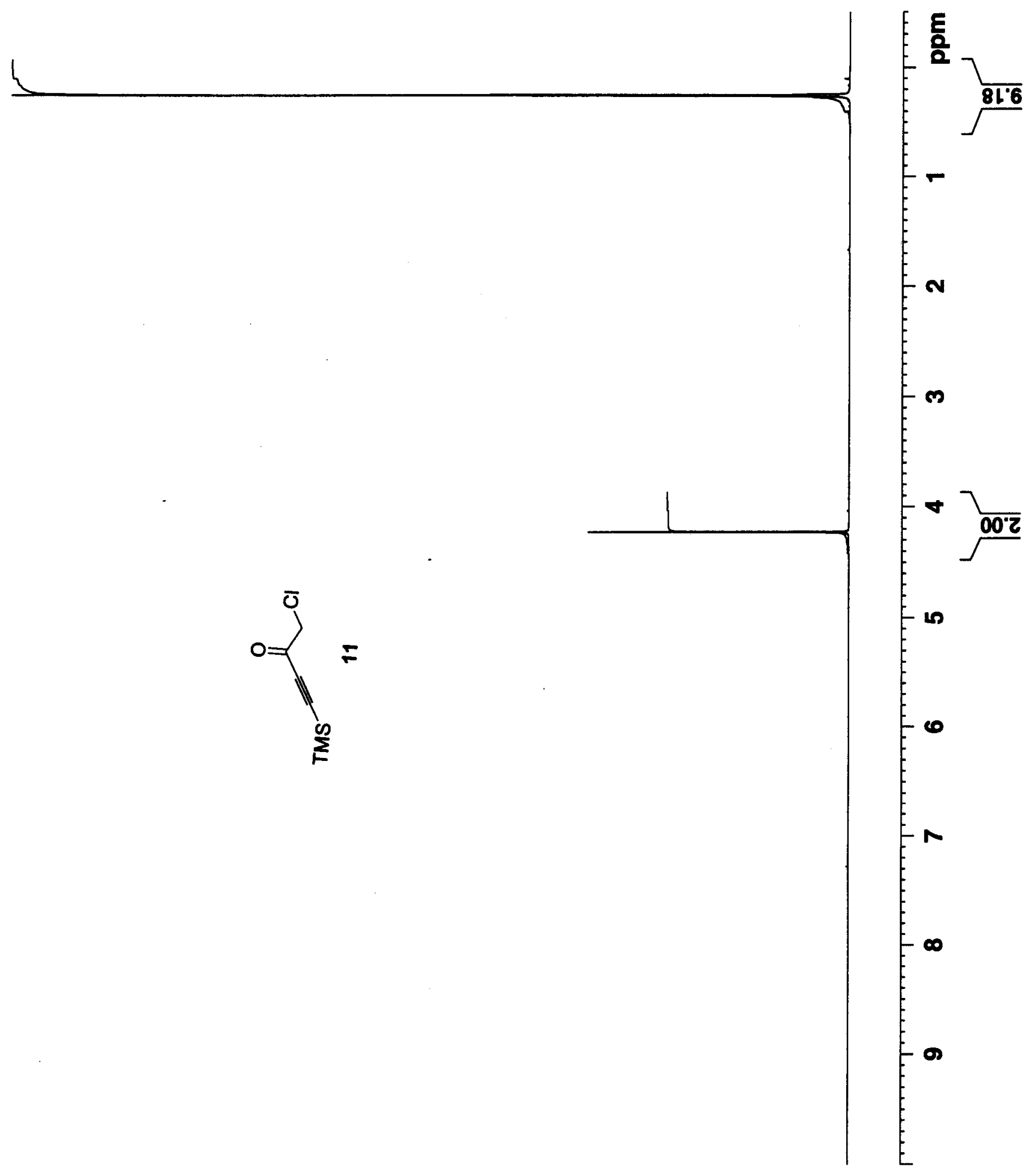

So 


\section{${ }^{13} \mathrm{C}$ NMR spectrum of compound 11}

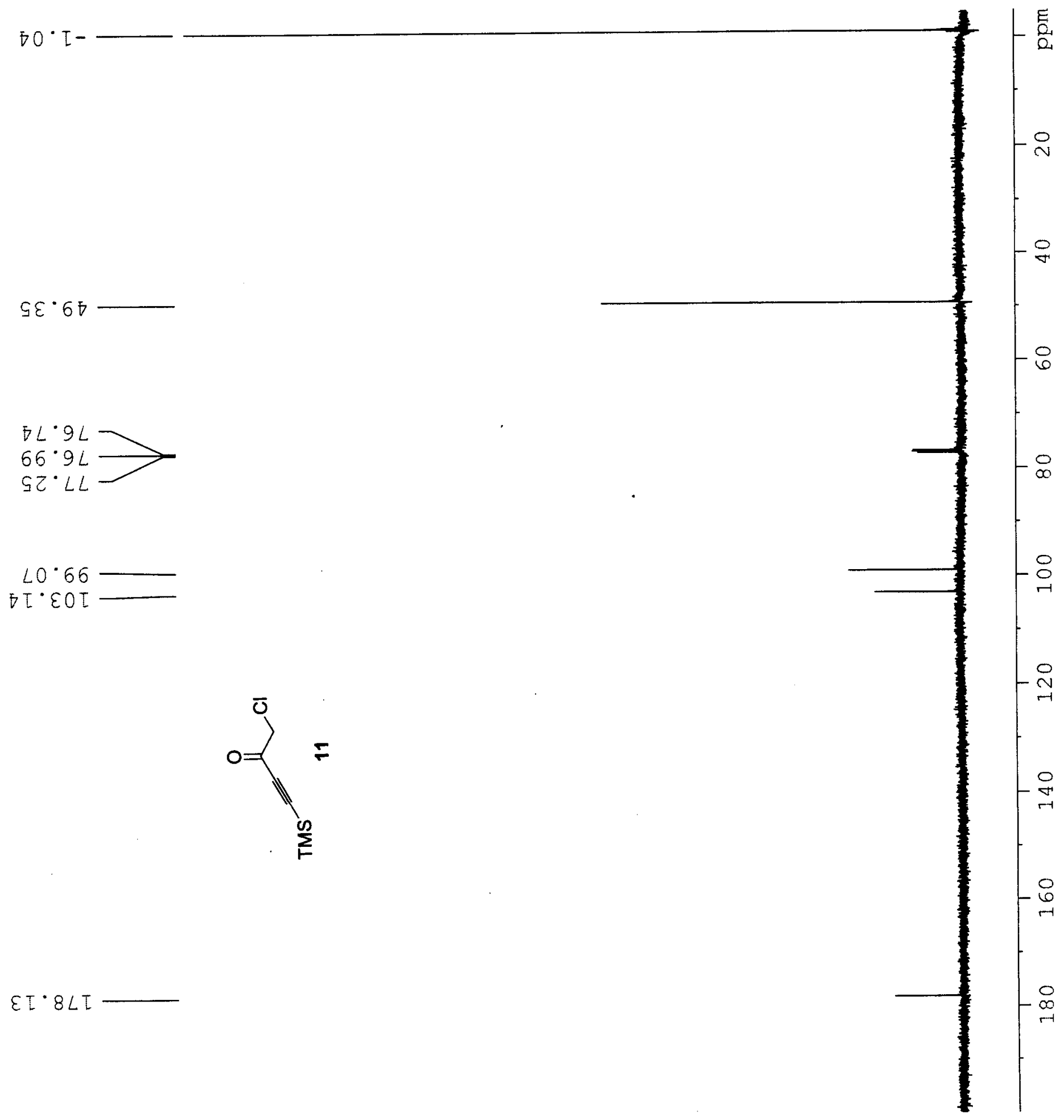


${ }^{1}$ H NMR spectrum of compound 9.

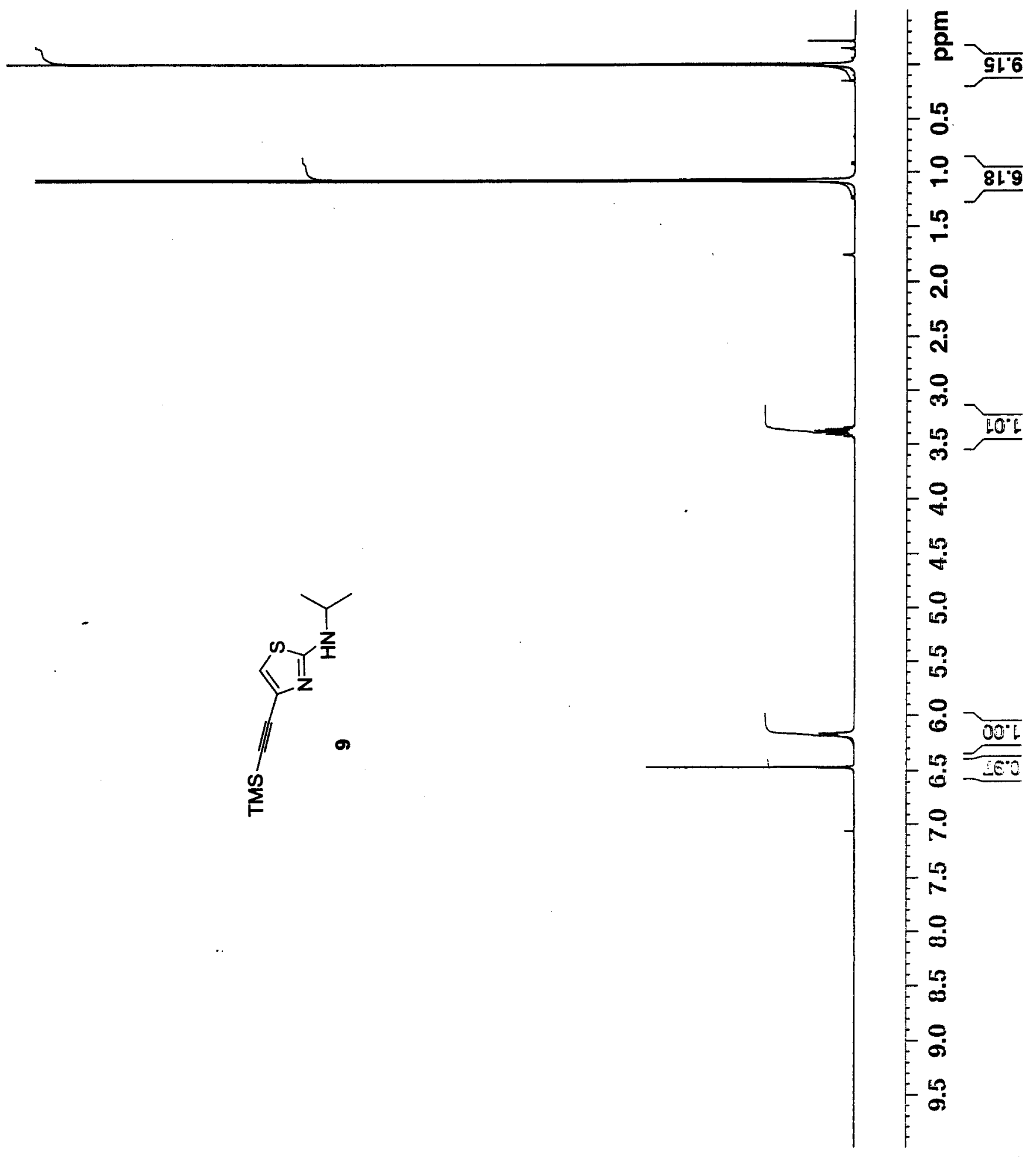




\section{${ }^{13} \mathrm{C}$ NMR spectrum of compound 9 .}

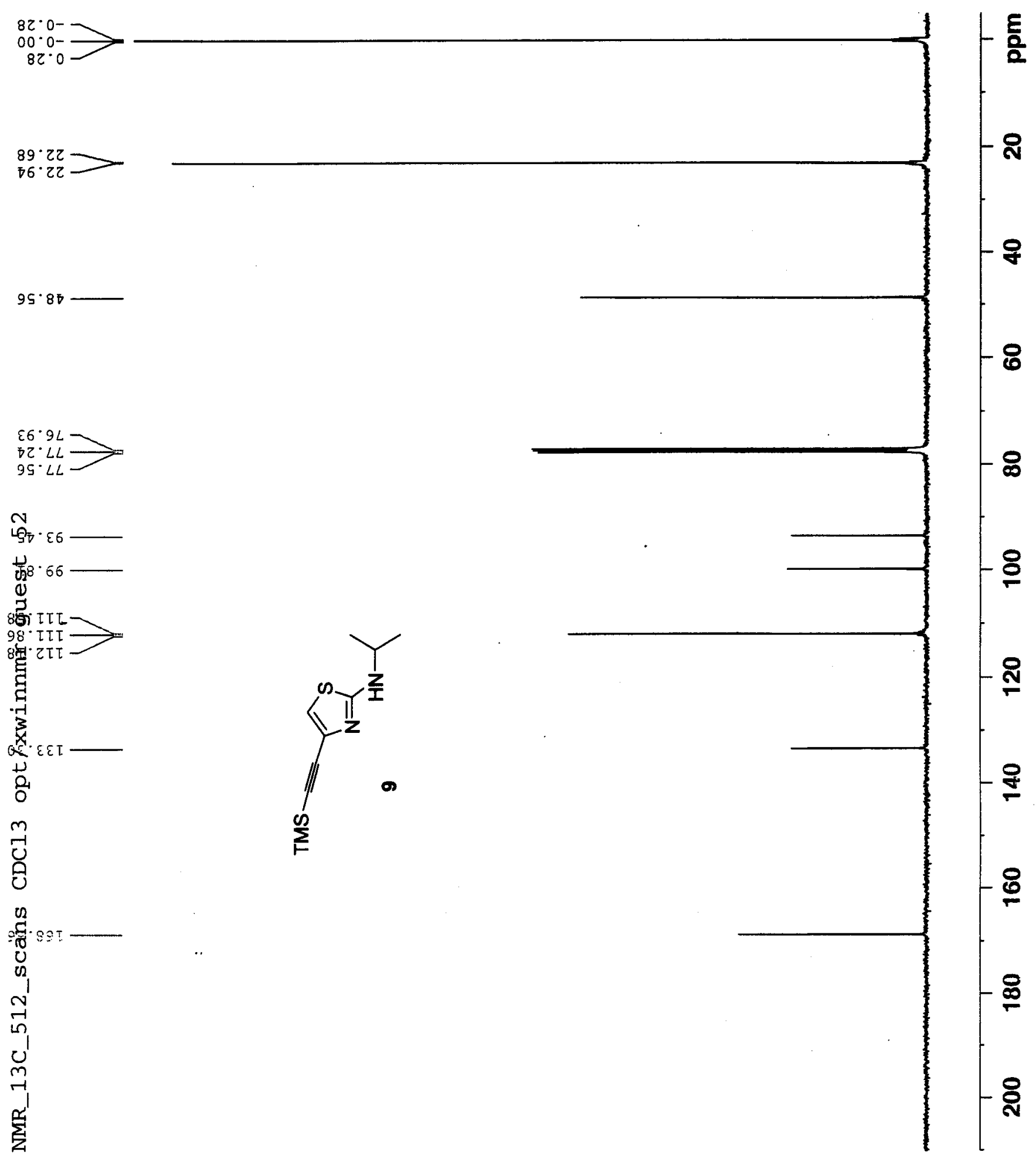


${ }^{1} \mathrm{H}$ NMR spectrum of compound 5 .

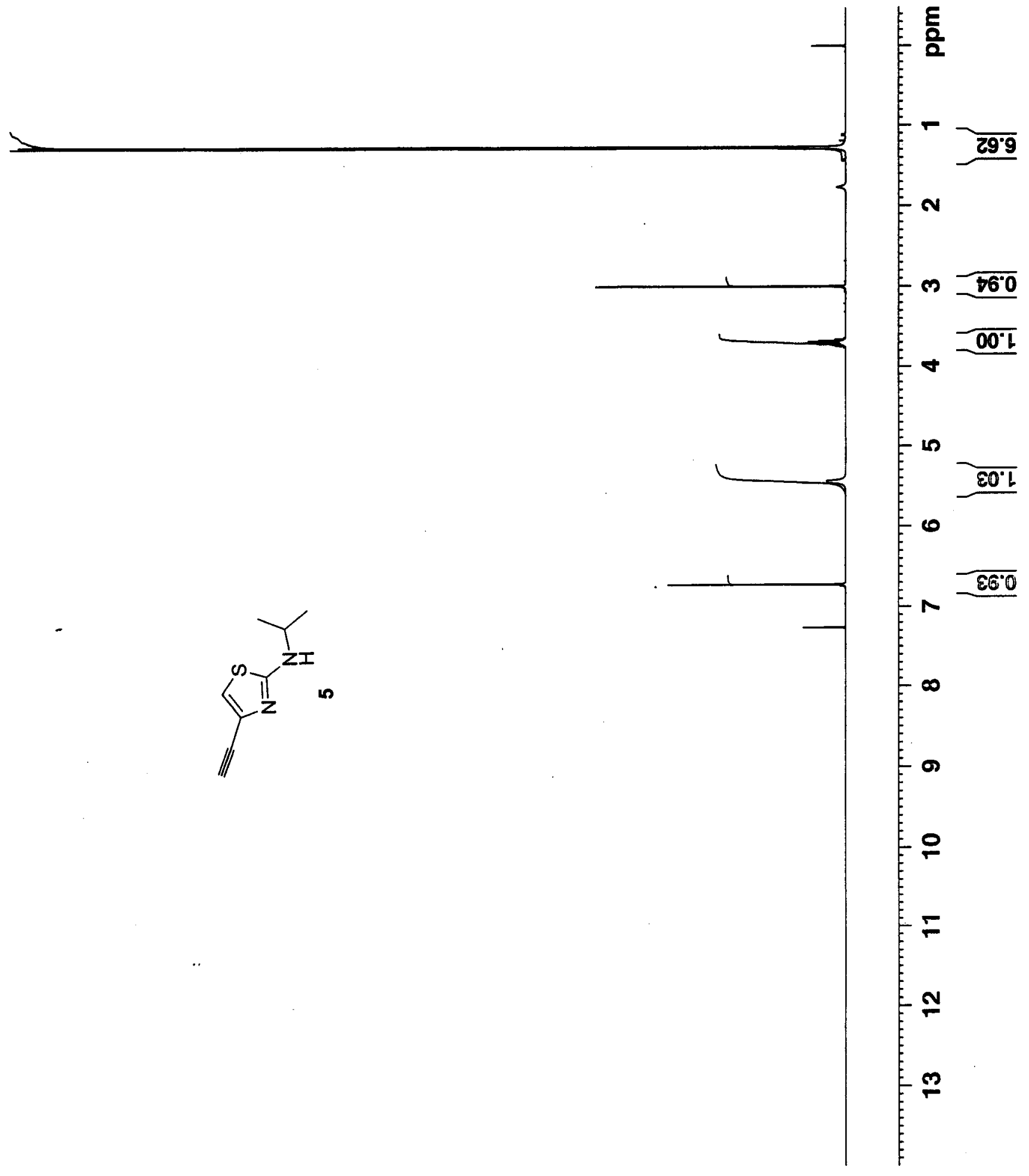


${ }^{13} \mathrm{C}$ NMR spectrum of compound 5.

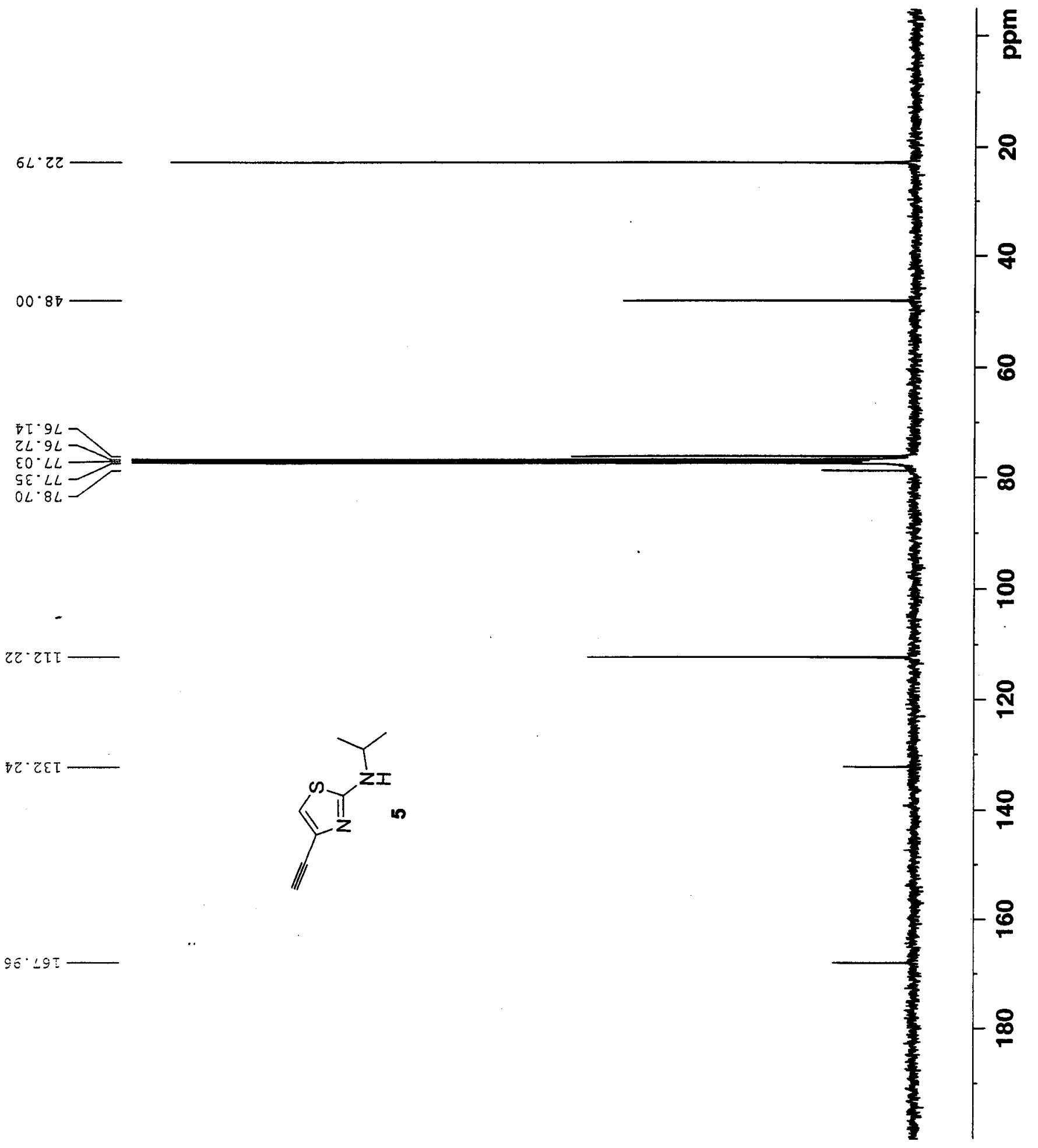


${ }^{1} \mathrm{H}$ NMR spectrum of compound 2.

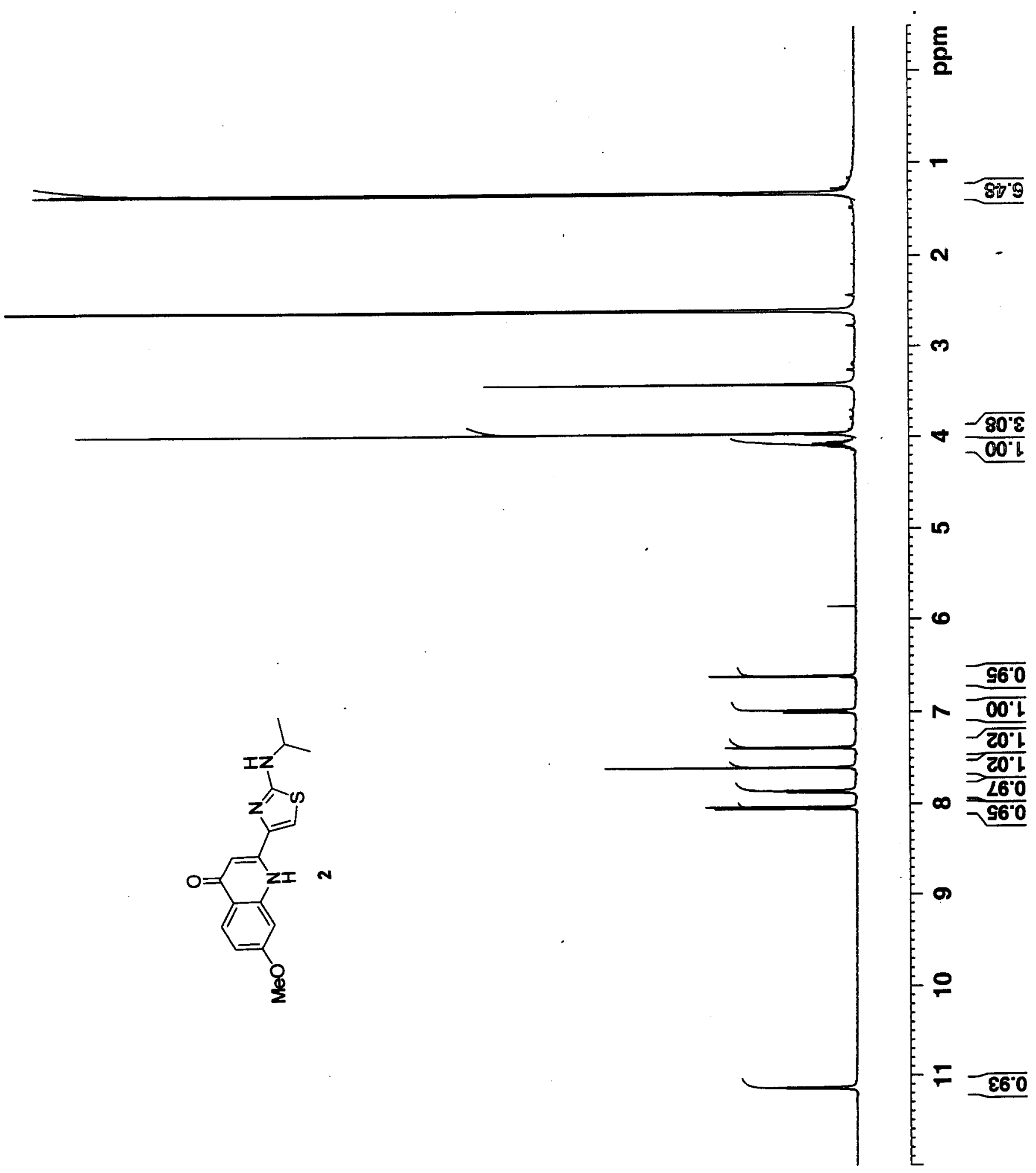


${ }^{1} \mathrm{H}$ NMR spectrum of compound 2 (Authentic Sample)

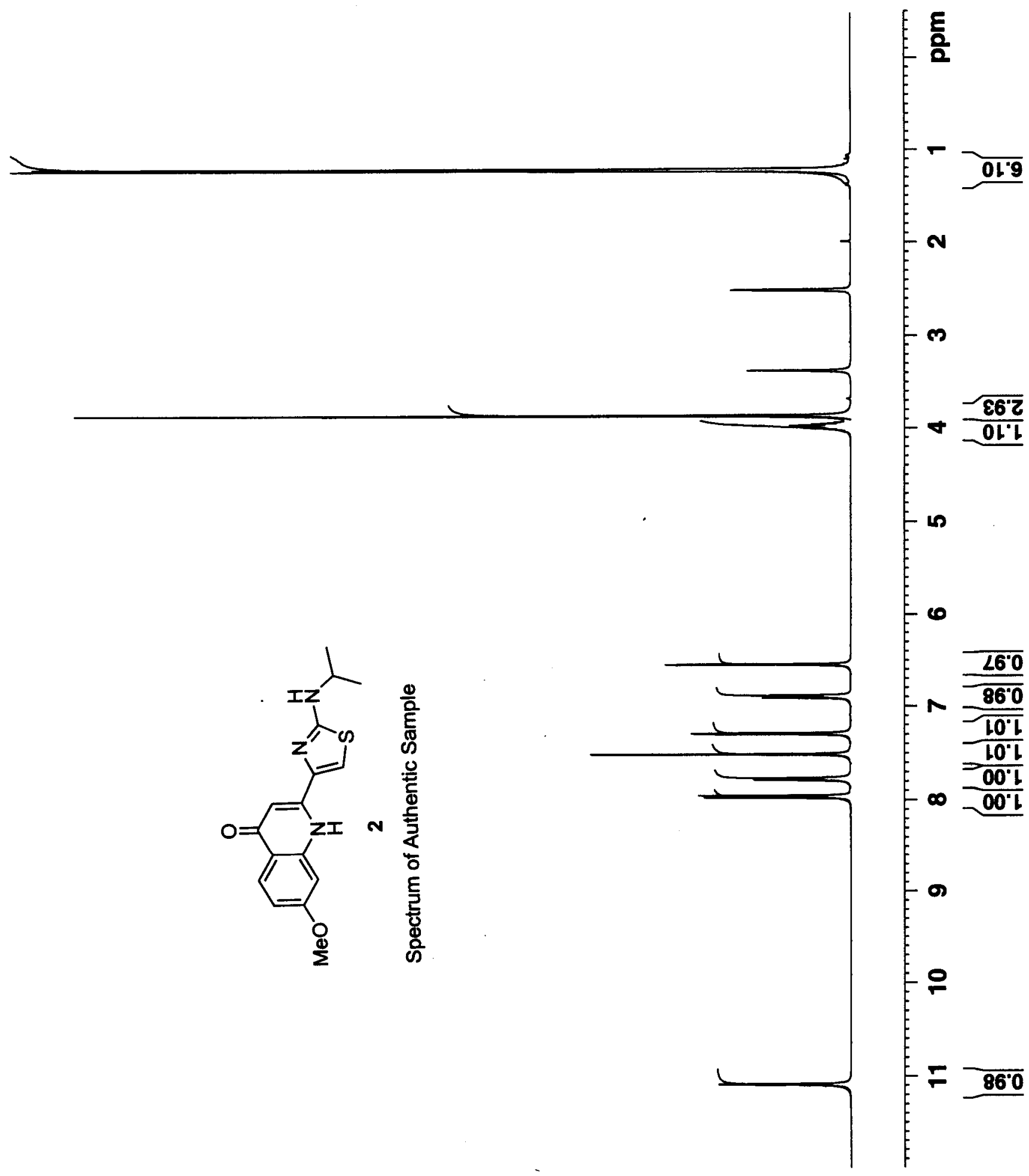


${ }^{13} \mathrm{C}$ NMR spectrum of compound 2.

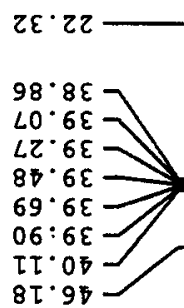

$8 T^{\circ} \cdot 97^{\circ}$

हธ. 55

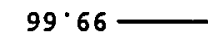

$89^{\circ} 901-$
$59.90 T=$

$\varepsilon 0^{\circ} \varepsilon \tau \tau \longrightarrow$

$\tau S \cdot 6 \tau \tau$

$\tau \cdot 9 \tau \tau$

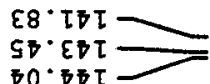
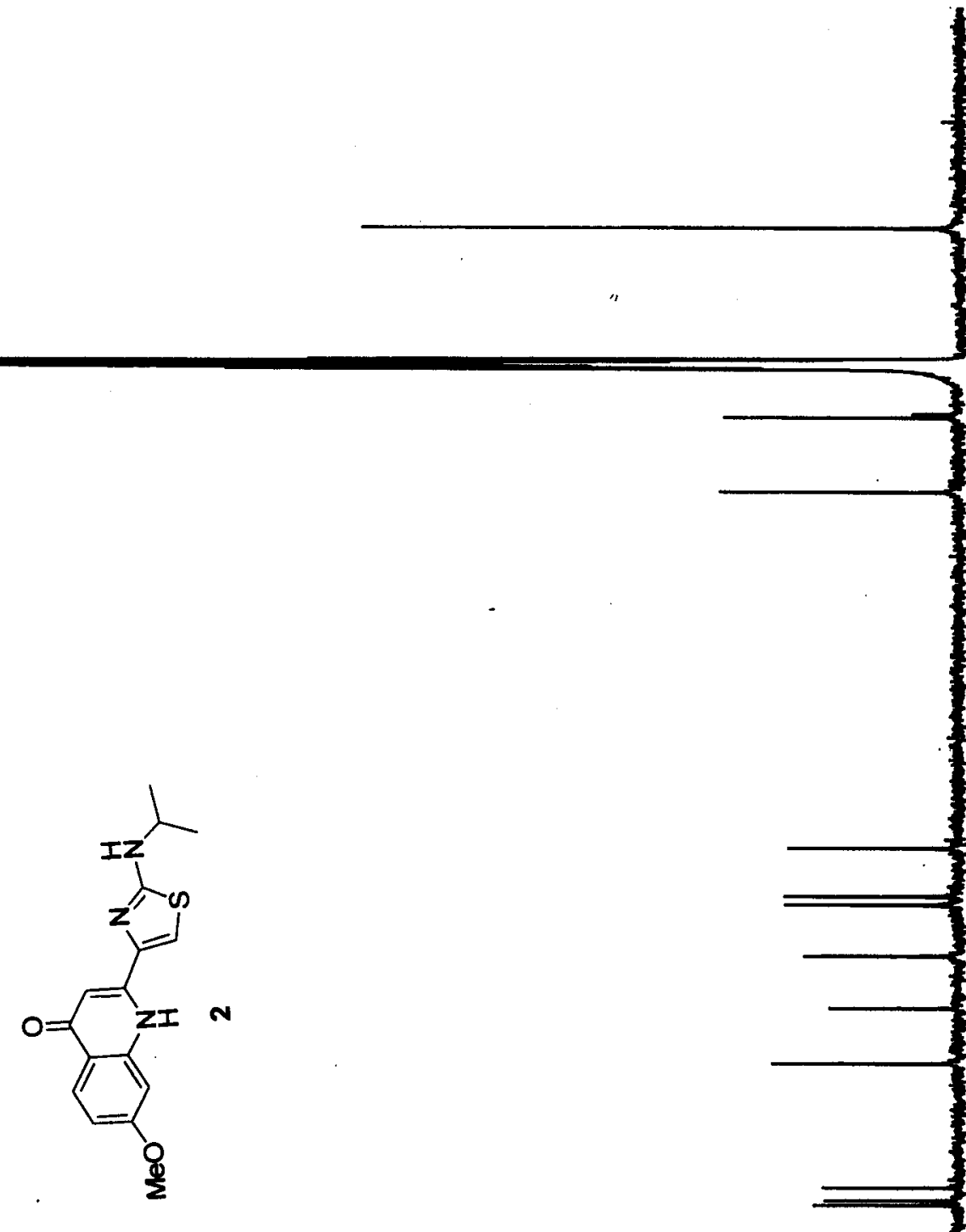

$-\frac{E}{2}$

$\frac{1}{2}$
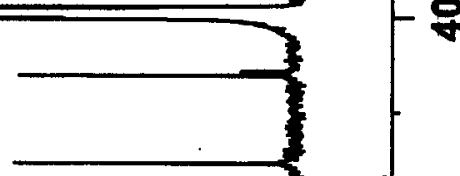

$06^{\circ}$ โ9โ

$6 S^{\circ} \angle 9 T-$

$\angle 9 \cdot 9 \angle \tau$

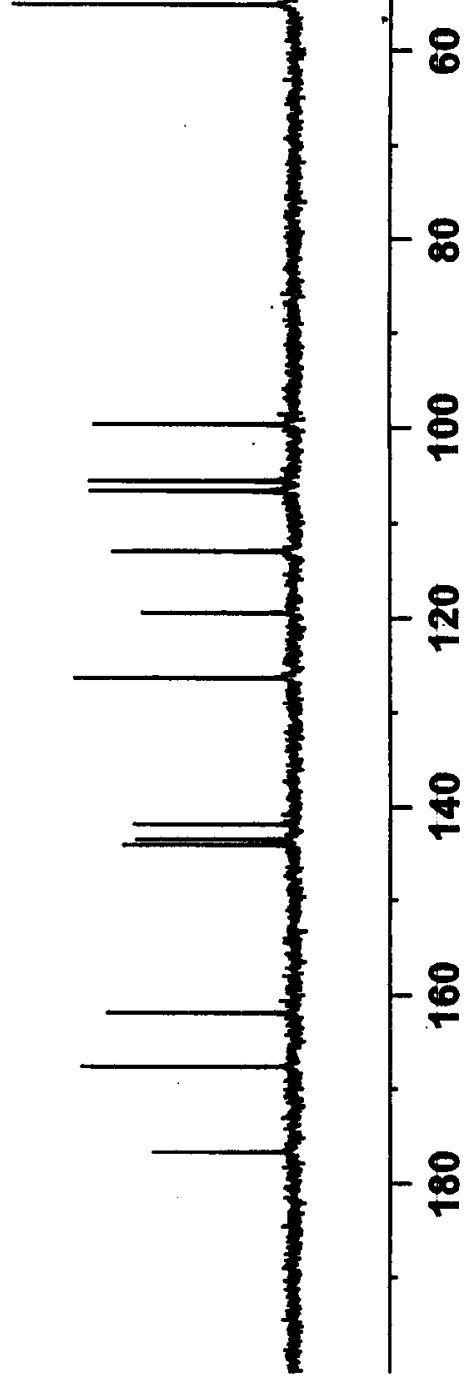


${ }^{13} \mathrm{C}$ NMR spectrum of compound 2. (Authentic Sample)

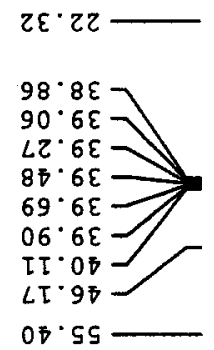

$59 \cdot 66$ $85 \cdot 50 \tau$
$29 \cdot 90 \tau$

โ० $\varepsilon \tau \tau$

$60 \cdot 6 \tau \tau$

$0 \overline{0} \cdot 9 Z T$

$\tau 8^{\circ} \tau B \tau$

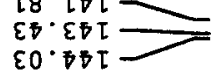

$88 \cdot \tau 9 \tau \longrightarrow$

$9 S \cdot \angle 9 I \longrightarrow$

$59 \cdot 9<\tau$

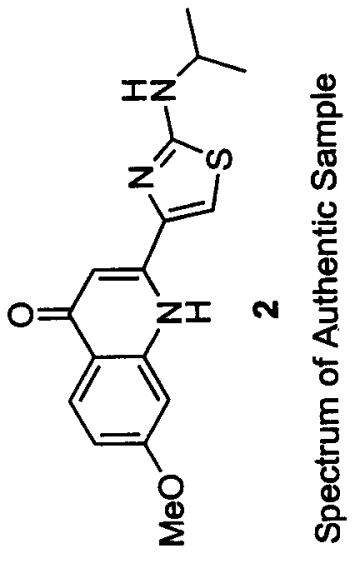

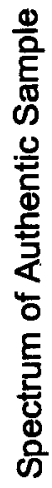

